\title{
ASOCIACIÓN ENTRE EL ESTADO DE INFECCIÓN POR VIH Y COMPLICACIONES INFECCIOSAS POSCESÁREA ELECTIVA
}

\author{
Romina Tejada1,2,a, Leandro Huayanay,b, César Gutiérrez¹,b, Carlos Velásquez ${ }^{4, c}$, César Loarte
}

RESUMEN

Objetivos. Determinar la asociación entre $\mathrm{VIH}$ y complicaciones infecciosas $(\mathrm{Cl})$ después de una cesárea electiva. Materiales y métodos. Estudio de cohortes no concurrentes; se revisaron las historias clínicas de 237 cesáreas electivas en gestantes VIH reactivas atendidas en el Instituto Nacional Materno Perinatal de Perú, realizadas entre 2004 a 2012. Estas fueron pareadas por cirujano y fecha de cesárea con 237 gestantes VIH no reactivas. Se recogieron datos sociodemográficos, clínicos, características de la cesárea y relacionados a la infección por el VIH. Las $\mathrm{Cl}$ se evaluaron según las recomendaciones del NHI Consensus Development Task Force. Se realizó el análisis bivariado con un nivel de significación del $5 \%$ y el cálculo del Odds Ratio (OR) con intervalo de confianza (IC) al 95\%. Se construyó un modelo de regresión logística condicional. Resultados. El 13,9\% de mujeres VIH positivas presentaron $\mathrm{Cl}$ y 9,7\% del grupo control (OR: 1,5 IC95\% [0,9-2,7]); las expuestas (mujeres con VIH) tuvieron mayor probabilidad de tener una infección del tracto urinario (ITU) que las no expuestas (ORa: 4,5 IC95\% [1,4-14,5]). Se encontró asociación entre $\mathrm{Cl}$ y el tipo de incisión (ORa: 2,3 IC95\% [1,1-4,5]) y el tiempo de hospitalización (6 frente a 3 días, $p<0,001)$. Conclusiones. Las mujeres $\mathrm{VIH}$ positivas sin tratamiento antirretroviral tuvieron mayor riesgo de $\mathrm{Cl}$; aunque no se observó mayor riesgo global de $\mathrm{Cl}$ en expuestas después de una cesárea electiva. El principal factor de riesgo asociado a una $\mathrm{Cl}$ poscesárea electiva en mujeres $\mathrm{VIH}$ positivas fue el tipo de incisión.

Palabras clave: VIH; Embarazo; Cesárea; Complicaciones postoperatorias; Complicaciones infecciosas del embarazo; (fuente: DeCS BIREME).

\section{ASSOCIATION BETWEEN HIV INFECTION STATUS AND INFECTIOUS COMPLICATIONS AFTER AN ELECTIVE C-SECTION}

\begin{abstract}
Objectives: To determine the connection between HIV and infectious complications (IC) after an elective C-section. Materials and methods. A non-concurrent cohort study was conducted, in which the clinical records of 237 elective C-sections on HIV-positive pregnant women who gave birth at the Instituto Nacional Materno Perinatal (National Maternal Perinatal Institute) between 2004 and 2012 were revised. The records were matched by surgeon and C-section date, with 237 HIV-negative pregnant women. Socio-demographic and clinical data were collected, along with characteristics of the C-section and data related to the HIV infection. IC were evaluated according to the NIH Consensus Development Task Force. The descriptive analysis and the bivariate analysis were carried out with a significance level of $5 \%$ for the hypothesis tests and the calculation of the Odds Ratio $(\mathrm{OR})$ with a confidence interval $(\mathrm{Cl})$ at $95 \%$. A conditional logistic regression model was built. Results. IC were present in $13.9 \%$ of HIV-positive women and $9.7 \%$ of the control group (OR: $1.5 \mathrm{Cl} 95 \%$ [0.9-2.7]); those who were exposed (women with HIV) were more likely to have a urinary tract infection (UTI) than those who were not exposed (ORa: $4.5 \mathrm{CI}$ \%5\% [1.4-14.5]). A connection was found between the IC and the type of incision (ORa: $2.3 \mathrm{Cl} 95 \%$ [1.1-4.5]) and time of hospitalization (6 versus 3 days, $p<0.001$ ). Conclusions. Exposed women did not register a greater global risk of IC after an elective C-section; however, they were, indeed, more likely to get a UTI. Those HIVpositive women who were not receiving antiretroviral treatment were in greater risk of IC. The main risk factors associated with a post C-section IC in HIV-positive women were the type of incision, as well as the surgery time with the UTI.
\end{abstract}

Key words: HIV; Pregnancy; Cesarean section; Postoperative complcation; Pregnancy complcation infections (source: MeSH NLM).

\footnotetext{
Sección Científica de Epidemiología, Instituto de Medicina Tropical, Universidad Nacional Mayor de San Marcos. Lima, Perú.

Unidad de Análisis y Generación de Evidencias en Salud Pública, Instituto Nacional de Salud. Lima, Perú.

Facultad de Medicina "Alberto Hurtado", Universidad Peruana Cayetano Heredia. Lima, Perú.

Instituto Nacional Materno Perinatal. Lima, Perú.

Médico cirujano, ${ }^{\mathrm{b}}$ médico cirujano magister en Epidemiología; ${ }^{\mathrm{c}}$ médico pediatra, ${ }^{\mathrm{d}}$ médico ginecólogo.

Recibido: 21-09-12 Aprobado: 14-11-12

* Los resultados provienen de la tesis para optar por el grado de magíster en Epidemiología Clínica realizada en la Universidad Peruana Cayetano Heredia.
}

Citar como: Tejada R, Huayanay L, Gutiérrez C, Velásquez C, Loarte C. Asociación entre el estado de infección por VIH y complicaciones infecciosas post cesárea electiva. Rev Peru Med Exp Salud Publica. 2012;29(4):444-51. 


\section{INTRODUCCIÓN}

Según datos de Programa Conjunto de las Naciones Unidas sobre el VIH/SIDA (ONUSIDA) el número estimado de personas que vivían con $\mathrm{VIH}$ en todo el mundo para el año 2009 alcanzó los 33,3 millones, siendo 2,6 millones de ellas nuevas infecciones; con un total de 1,8 millones de defunciones ${ }^{(1)}$. En el caso del Perú, hasta julio de 2012, se habían notificado 47469 casos de infección por VIH y 29297 de SIDA (2).

En nuestro país la epidemia se encuentra concentrada en el grupo de hombres que tienen sexo con otros hombres; sin embargo, se ha ido extendiendo progresivamente a la población heterosexual; es así que, desde finales de los años 90 , las mujeres representan aproximadamente el $25 \%$ de la población infectada (razón hombre/mujer de $3 / 1$ en 2011) (2). Dicho incremento trae a discusión el problema de la transmisión vertical del $\mathrm{VIH}$, siendo la prevalencia en gestantes, según vigilancia centinela de $0,2 \%$ para el $2006^{(3)}$.

La tasa de transmisión del VIH de la madre al niño puede ser notablemente reducida, hasta menos del $1 \%$, con una serie de medidas entre ellas el parto por cesárea electiva, previa al inicio de trabajo de parto o ruptura de membranas ${ }^{(4-5)}$. Así, por ejemplo, un estudio colaborativo europeo ${ }^{(6)}$ encontró que la cesárea sin ninguna otra intervención disminuía la transmisión vertical (TV) hasta en un $50 \%$. Debemos también considerar que, al prevenir la transmisión vertical del VIH, en EE. UU. se ha estimado que la cesárea electiva logra un ahorro de al menos 5,3 millones de dólares al año ${ }^{(7)}$.

Es así que la cesárea electiva ha pasado a formar parte de las normas nacionales ${ }^{(8)}$ para la prevención de la TV del VIH. Sin embargo, con el aumento del uso y efectividad del Terapia Antirretroviral de Gran Actividad, logrando cargas virales maternas menores de mil copias al momento del parto, se ha vuelto difícil discernir si las ventajas de cesáreas electivas compensan sus riesgos ${ }^{(9)}$.

Es ampliamente aceptado que la cesárea, tanto en personas infectadas con el $\mathrm{VIH}$ como en las que no, conlleva una serie de complicaciones en comparación con el parto vaginal; siendo las complicaciones infecciosas las más comunes. Entre ellas tenemos a la endometritis, infección del tracto urinario (ITU) e infección de herida operatoria $(\mathrm{IHO})^{(10,11)}$. Podríamos suponer que, debido a su estado de infección, las mujeres $\mathrm{VIH}$ reactivas tendrían un mayor riesgo de infecciones posoperatorias. Sin embargo, los datos de estudios realizados al respecto son aún controversiales ${ }^{(12-15)}$. Uno de los principales estudios que comparó las diferencias en la tasa de complicaciones entre mujeres con y $\sin \mathrm{VIH}$ sometidas a cesárea electiva, observó un aumento en la frecuencia de complicaciones generales en el grupo de mujeres con $\mathrm{VIH}^{(12)}$. Sin embargo, también hay estudios que han observado frecuencias similares de complicaciones en ambos grupos (16,17). Esto nos trae la necesidad de evaluar las potenciales complicaciones infecciosas poscesárea en mujeres VIH positivas comparadas con mujeres seronegativas.

No podemos dejar de considerar que las gestantes HIV reactivas son una población especial, siendo muchas veces diagnosticadas durante sus controles prenatales, en estadios tempranos y relativamente asintomáticas, pero con muy variados estados inmunológicos y en las cuales, si bien se realiza una cesárea electiva, esta no puede retrasarse hasta una mejoría del estado de salud. Por lo antes mencionado fue objetivo del presente estudio comparar la tasa de complicaciones infecciosas posoperatorias en mujeres $\mathrm{VIH}$ positivas intervenidas por cesárea electiva, frente a gestantes $\mathrm{VIH}$ negativas, en el Instituto Nacional Materno Perinatal (INMP).

\section{MATERIAL Y MÉTODOS}

\section{DISEÑO Y POBLACIÓN}

Se realizó un estudio de cohortes no concurrentes, en el cual se revisaron las historias clínicas de dos grupos de mujeres a quienes se les realizó cesárea electiva entre enero de 2004 y febrero de 2012 en el INMP, diferenciadas por su estado de infección por el VIH, documentado por pruebas de ELISA y Western Blot o que cumplieran con los criterios clínicos de la Estrategia Sanitaria Nacional de Prevención y Control de las ITS, VIH y SIDA. El grupo de mujeres expuestas estuvo conformado por mujeres $\mathrm{VIH}$ positivas las cuales fueron identificadas en el registro del Servicio de Medicina Preventiva del INMP, otorgado por uno de los autores (CV). Posteriormente, se buscó en el registro de cesáreas del INMP a las mujeres que fueron operadas por el mismo cirujano en un periodo de dos días antes o después de la fecha de cesárea del par expuesto. Se excluyeron mujeres con trastornos por inmunodeficiencia heredados o por tratamiento, con ruptura prematura de membranas, y parto gemelar. En el caso de que una de las participantes presentara dos cesáreas dentro del lapso de estudio, cada episodio fue considerado de manera independiente. Se realizó un muestreo no probabilístico por conveniencia solicitando las historias clínicas, desde las más recientes hacia las más antiguas, hasta completar el tamaño muestral.

\section{TAMAÑO MUESTRAL}

Se calculó un tamaño muestral de 237 cesáreas por grupo considerando las tasas reportadas por Fiore et al. ${ }^{(14)}$ de 
$30 \%$ en infectadas y $18 \%$ en no infectadas con $\mathrm{VIH}$, una relación 1:1 entre grupos, un nivel de significancia del 95\%, un poder de $80 \%$ y un $10 \%$ de no respuesta; empleando la función para cálculo de tamaño muestral en estudios de cohortes del paquete estadístico Epidat.

\section{RECOLECCIÓN DE LOS DATOS}

En una ficha adhoc se recogieron datos de variables sociodemográficas (edad, grado de instrucción, estado civil y ocupación); clínicas (estado de infección por el VIH, estado nutricional, consumo de tabaco y otras drogas) y características de la cesárea (número de partos, cirujano, indicación principal de la cesárea, tipo de incisión, líquido amniótico, tiempo operatorio, tiempo de hospitalización poscesárea, uso de antibióticos y uso de catéter urinario). En las mujeres $\mathrm{VIH}$ positivas se recogieron además variables relacionadas a la infección por el VIH (tiempo de diagnóstico de VIH, carga viral, conteo de CD4, estadio clínico e inmunológico según OMS y uso de TARGA al momento de la evaluación postparto).

Los datos de complicaciones infecciosas fueron recogidos según las recomendaciones para evaluación de morbilidad poscesárea del NHI Consensus Development Task Force ${ }^{(18)}$. Es así que se definió complicación infecciosa poscesárea como la presencia de uno o más de los siguientes: endometritis, infección de herida operatoria, fiebre sin foco aparente de infección o infección de tracto urinario; que se presentara hasta 42 días posteriores a la cesárea, según reporte en las visitas de seguimiento poscesárea.

\section{PROCESAMIENTO Y ANÁLISIS DE DATOS}

Los datos fueron ingresados a una base de datos en el programa STATA versión 10.0; se realizó un control de calidad basado en la revisión periódica del $10 \%$ de las fichas digitadas (de forma aleatoria). El análisis estadístico consistió en una primera etapa de análisis descriptivo, obteniendo medidas de resumen y distribuciones de frecuencia, así como la proporción de mujeres con complicaciones infecciosas en cada grupo, con un intervalo de confianza de 95\%. El análisis bivariado consistió en la determinación de la asociación entre las variables del estudio mediante la prueba chicuadrado o Fisher para las variables categóricas cualitativas según correspondiera; y la prueba de $t$ de Student o $U$ de Mann-Whitney para las variables cuantitativas; se usó un nivel de significación del $5 \%$. También se calculó como medida de fuerza de asociación el OR con intervalos de confianza de 95\%. Finalmente se construyó un modelo de regresión logística condicional stepwise backward para el cálculo de los OR ajustados; se consideró incluir aquellas variables que tuvieran significancia estadística en el análisis bivariado o que los investigadores consideraran pertinentes en base al marco lógico.

\section{ASPECTOS ÉTICOS}

El presente estudio fue revisado y aceptado por el Comité de Ética del Instituto Nacional Materno Perinatal y de la Universidad Peruana Cayetano Heredia, y corresponde a un estudio de mínimo riesgo ya que no hubo contacto con los participantes y la información fue manejada con códigos y exclusivamente por los autores.

\section{RESULTADOS}

Se revisaron 217 historias clínicas de mujeres VIH reactivas, se excluyeron ocho debido a que la indicación de la cesárea se rotuló como sufrimiento fetal agudo; en 26 casos las mujeres habían tenido dos cesáreas durante el periodo de estudio y en un caso tres cesáreas, lo cual nos dio una muestra final de 237 cesáreas en mujeres $\mathrm{VIH}$ reactivas. De igual manera, se revisaron 214 historias clínicas de mujeres VIH no reactivas, se excluyeron tres debido a que fueron mal rotuladas como electivas, siendo el motivo de la cesárea sufrimiento fetal en dos casos y desprendimiento de placenta en uno; además, 24 de ellas tuvieron dos cesáreas en el periodo de estudio y una tres cesáreas, lo que nos dio una muestra final de 237 cesáreas en mujeres $\mathrm{VIH}$ no reactivas. En todos los casos la cesárea fue a término y previo a la ruptura de membranas; además ninguna de las mujeres estaba en trabajo de parto. Todas las cesáreas fueron realizadas por un médico asistente y bajo anestesia epidural o espinal.

Las mujeres expuestas y no expuestas fueron similares en edad (mediana de 28 años para ambos grupos), consumo de tabaco, estado nutricional, tipo de líquido amniótico, uso de catéter urinario y uso de antibióticos $(p>0,05)$. El tiempo operatorio también fue similar con 36 minutos (rango intercuartil 27-47) para las expuestas y 35 minutos (rango intercuartil 27-44) en el caso de las no expuestas. Por otro lado los grupos diferían en su proporción de mujeres unidas, en su grado de instrucción, ocupación, consumo de drogas, número de partos, tipo de incisión e indicación de la cesárea. Entre las indicaciones de cesárea, el motivo más frecuente en las mujeres expuestas fue evitar la TV (73\%); mientras que en el grupo de no expuestas fue la incompatibilidad céfalo-pélvica (por macrosomía fetal o por estrechez pélvica). El tiempo de hospitalización posoperación también fue mayor en el grupo de mujeres VIH reactivas $(p=0,028)$ (Tabla 1$)$.

Entre las mujeres que tenían diagnóstico de $\mathrm{VIH}$ se observó que el tiempo de diagnóstico del VIH era en su mayoría menor a un año (mediana de 8,3 meses, 
Tabla 1. Características sociodemográficas y clínicas de gestantes a quienes se realizó cesárea electiva en el INMP, 2004-2012.

\begin{tabular}{|c|c|c|c|}
\hline & $\begin{array}{c}\mathrm{VIH} \\
\mathrm{N} .{ }^{\circ}(\%) \\
\end{array}$ & $\begin{array}{l}\text { No VIH } \\
\text { N. }{ }^{\circ}(\%) \\
\end{array}$ & $\begin{array}{c}\text { Valor } \\
p^{*}\end{array}$ \\
\hline \multicolumn{4}{|l|}{ Estado civil } \\
\hline Unido & $192(81,4)$ & $211(89,0)$ & 0,019 \\
\hline No unido & $44(18,6)$ & $26(0,1)$ & \\
\hline \multicolumn{4}{|l|}{ Grado de instrucción } \\
\hline Analfabeto & $2(0,8)$ & $1(0,4)$ & 0,005 \\
\hline Primaria & $29(12,2)$ & $15(6,3)$ & \\
\hline Secundaria & $179(75,5)$ & $171(72,1)$ & \\
\hline Superior & $27(11,4)$ & $50(21,1)$ & \\
\hline \multicolumn{4}{|l|}{ Ocupación } \\
\hline Ama de casa & $206(86,9)$ & $186(78,5)$ & 0,005 \\
\hline Empleado tiempo completo & $8(3,5)$ & $27(11,4)$ & \\
\hline Empleado tiempo parcial & $18(7,8)$ & $21(8,9)$ & \\
\hline Estudiante & $5(2,2)$ & $3(1,3)$ & \\
\hline $\begin{array}{l}\text { Consumo } \\
\text { de vida de tabaco }\end{array}$ & $9(3,9)$ & $3(1,4)$ & 0,142 \\
\hline $\begin{array}{l}\text { Consumo } \\
\text { de vida de drogas }\end{array}$ & $14(6,3)$ & $3(1,4)$ & 0,011 \\
\hline \multicolumn{4}{|c|}{ Estado nutricional pregestación } \\
\hline Desnutrido & $17(7,9)$ & $15(6,9)$ & 0,477 \\
\hline Eutrófico & $110(50,9)$ & $96(44,4)$ & \\
\hline Sobrepeso & $67(31,0)$ & $77(35,7)$ & \\
\hline Obeso & $22(10,2)$ & $28(13,0)$ & \\
\hline Incisión Media Infraumbilical & $39(16,8)$ & $23(9,7)$ & 0,029 \\
\hline Multipara & $69(29,5)$ & $36(15,4)$ & $\leq 0,001$ \\
\hline \multicolumn{4}{|l|}{ Indicación de la cesárea } \\
\hline Prevención de la TV & $171(73,1)$ & $0(0,0)$ & $\leq 0,001$ \\
\hline Cesárea previa & $32(13,7)$ & $48(20,5)$ & \\
\hline Complicación materna & $11(4,7)$ & $38(16,2)$ & \\
\hline Mal posición fetal & $9(3,9)$ & $41(17,5)$ & \\
\hline $\begin{array}{l}\text { Incompatibilidad } \\
\text { céfalo-pélvica }\end{array}$ & $3(1,3)$ & $58(24,8)$ & \\
\hline Distocia funicular & $4(1,7)$ & $33(14,1)$ & \\
\hline Deseo materno & $0(0,0)$ & $5(2,1)$ & \\
\hline Otro & $7(3,0)$ & $14(6,0)$ & \\
\hline $\begin{array}{l}\text { Líquido } \\
\text { amniótico meconial }\end{array}$ & $26(11,0)$ & $37(15,6)$ & 0,137 \\
\hline Uso de catéter urinario & $165(98,2)$ & $171(97,7)$ & 0,743 \\
\hline Uso de antibióticos & $187(78,9)$ & $186(78,5)$ & 0,911 \\
\hline
\end{tabular}

rango intercuartil: 2,4-40,3 meses). Se contó con datos de carga viral en 117 mujeres, se observó que el 32,5\% tenía carga viral detectable (por encima de 400 copias/ $\mathrm{mL}$ ); de igual modo, se observó que la mediana del conteo de CD4 fue $347,5 \mathrm{cel} / \mathrm{mL}$ (rango intercuartil: $235,5-580,0 \mathrm{cel} / \mathrm{mL}$ ). En relación a la categoría clínica de la OMS la mayoría se encontraba en estadio $1(90,1 \%)$. Por otro lado, el $31,5 \%$ se encontraba en un estadio inmunológico de la OMS leve. El $86 \%$ de las gestantes VIH positivas había recibido ARV (Antirretroviral) durante la gestación o periparto, con una mediana de tiempo
Tabla 2. Características clínicas de las pacientes $\mathrm{VIH}$ reactivas a quienes se realizó cesárea electiva. INMP, 2004-2012.

\begin{tabular}{|c|c|}
\hline & N. ${ }^{\circ}(\%)$ \\
\hline Carga viral detectable $(n=117)$ & $38(32,5)$ \\
\hline \multicolumn{2}{|l|}{ Categoría clínica OMS (n=211) } \\
\hline 1 & $190(90,1)$ \\
\hline 2 & $6(2,8)$ \\
\hline 3 & $7(3,3)$ \\
\hline 4 & $8(3,8)$ \\
\hline \multicolumn{2}{|l|}{ Categoría inmunológica OMS $(\mathrm{n}=111)$} \\
\hline Leve $(\geq 500 \mathrm{cel} / \mathrm{ml})$ & $35(31,5)$ \\
\hline Moderada $(350-499 \mathrm{cel} / \mathrm{ml})$ & $16(14,4)$ \\
\hline Avanzada (200-349 cel/ml) & $28(25,2)$ \\
\hline $\operatorname{SIDA}(<200 \mathrm{cel} / \mathrm{ml})$ & $32(28,8)$ \\
\hline Uso de ARV $(n=235)$ & $202(86,0)$ \\
\hline \multicolumn{2}{|l|}{ Última vez que perdió una dosis* $(n=78)$} \\
\hline Nunca & $37(47,4)$ \\
\hline Durante las últimas dos semanas & $6(7,7)$ \\
\hline Durante el último mes & $15(19,2)$ \\
\hline Hace más de un mes & $20(25,6)$ \\
\hline
\end{tabular}

en TARGA de 3,84 meses (rango intercuartil 1,5-15,8 meses); entre estas se contó con información de pérdida de dosis de ARV en 78 mujeres, 37 de ellas informaron $(47,4 \%)$ no haber perdido nunca una dosis (Tabla 2 ).

La incidencia acumulada de complicaciones infecciosas (Cl) fue de $13,9 \%$ (33) en el caso de las mujeres VIH positivas y de $9,7 \%$ (23) en las controles; dicha diferencia no fue significativa (OR: 1,5 IC95\% [0,9-2,7]). Entre las mujeres $\mathrm{VIH}$ positivas se observaron 12 casos $(5,1 \%)$ de infecciones de herida operatoria, igual número en las controles; por otro lado, en las expuestas hubo 17 casos $(7,2 \%)$ de infección del tracto urinario, mientras que en las no expuestas se registraron solo seis casos (OR: 3,0; IC95\% [1,2-7,7]). Hubieron dos casos de endometritis entre las mujeres $\mathrm{VIH}$ positivas y tres entre las negativas (OR: 0,7; IC95\% [0,1-4,0]). Finalmente, se observaron siete casos $(3 \%)$ de fiebre sin foco aparente entre las mujeres $\mathrm{VIH}$ positivas y cuatro casos $(1,7 \%)$ en las negativas (OR: 1,8; IC95\% [0,5-6,1]).

Entre los otros factores asociados a complicaciones infecciosas (Tabla 3), en los dos grupos, se encontró que las mujeres con incisión tipo media infraumbilical (MIU) tenían mayor probabilidad de presentar una $\mathrm{Cl}$ en comparación con las mujeres a las que se les hizo una incisión tipo Phannesteil (OR: 2,3; IC 95\% [1,1-4,5]). También se observó que las mujeres con una educación primaria o inferior tenían mayor riesgo de presentar una $\mathrm{Cl}$ que las que tenía educación secundaria o superior (OR: 2,2; IC 95\% [1,0-4,8]). Como era de esperar se encontró que el tiempo de hospitalización poscesárea fue mayor en el grupo de mujeres con $\mathrm{Cl}$ (mediana seis 
Tabla 3. Factores asociados a complicación infecciosa poscesárea electiva en el INMP, 2004-2012.

\begin{tabular}{|c|c|c|c|c|}
\hline & \multicolumn{2}{|c|}{$\begin{array}{c}\text { Complicación } \\
\text { infecciosa }\end{array}$} & \multirow[t]{2}{*}{ OR } & \multirow[t]{2}{*}{ [IC 95\%] } \\
\hline & Sí & No & & \\
\hline \multicolumn{5}{|l|}{ Estado de infección } \\
\hline VIH (+) & 33 & 204 & 1,51 & {$[0,85-2,65]$} \\
\hline VIH (-) & 23 & 214 & 1 & \\
\hline \multicolumn{5}{|l|}{ Estado civil } \\
\hline No unida & 13 & 57 & 1,91 & {$[0,97-3,77]$} \\
\hline Unida & 43 & 360 & 1 & \\
\hline \multicolumn{5}{|l|}{ Grado de instrucción } \\
\hline Primaria o inferior & 10 & 37 & 2,24 & {$[1,04-4,80]$} \\
\hline Secundaria/Superior & 46 & 381 & 1 & \\
\hline \multicolumn{5}{|l|}{ Consumo de tabaco } \\
\hline Sí & 2 & 10 & 1,50 & {$[0,32-7,02]$} \\
\hline No & 50 & 374 & 1 & \\
\hline \multicolumn{5}{|l|}{ Consumo de drogas } \\
\hline Sí & 2 & 15 & 0,98 & {$[0,22-4,41]$} \\
\hline No & 50 & 367 & 1 & \\
\hline \multicolumn{5}{|l|}{ Estado nutricional } \\
\hline Desnutrido & 3 & 29 & 0,78 & {$[0,22-2,77]$} \\
\hline Eutrófico & 24 & 182 & 1 & \\
\hline Sobrepeso & 15 & 129 & 1,88 & {$[0,44-1,75]$} \\
\hline Obeso & 5 & 45 & 0,84 & {$[0,30-2,26]$} \\
\hline \multicolumn{5}{|l|}{ Uso de catéter urinario } \\
\hline Sí & 30 & 306 & 0,24 & {$[0,05-1,32]$} \\
\hline No & 2 & 5 & 1 & \\
\hline \multicolumn{5}{|l|}{ Tipo de incisión } \\
\hline Media infraumbilical & 13 & 49 & 2,28 & {$[1,14-4,53]$} \\
\hline Pfannesteil & 43 & 369 & 1 & \\
\hline \multicolumn{5}{|l|}{ Uso de antibióticos } \\
\hline No & 8 & 93 & 0,58 & {$[0,27-1,27]$} \\
\hline Sí & 48 & 325 & 1 & \\
\hline \multicolumn{5}{|l|}{ Multipara } \\
\hline Sí & 14 & 91 & 1,20 & {$[0,63-2,29]$} \\
\hline No & 42 & 327 & 1 & \\
\hline \multicolumn{5}{|l|}{ Líquido amniótico } \\
\hline Meconial & 7 & 56 & 0,92 & {$[0,40-2,14]$} \\
\hline Claro & 49 & 362 & 1 & \\
\hline
\end{tabular}

días; rango intercuartil: 3-9 días) que en el grupo de mujeres sin complicación infecciosa (mediana tres días; rango intercuartil: 3-4 días, $p<0,001)$. No se encontró asociación con la edad, ni con el tiempo operatorio.

Además de estar asociada al estado de infección por el $\mathrm{VIH}$, la infección del tracto urinario estuvo asociada a un mayor tiempo operatorio $(p=0,03)$, teniendo el grupo que padeció ITU 43 minutos (rango intercuartil 30-51 minutos) y las que no 36 minutos (rango intercuartil 27-45 minutos). De igual manera las mujeres que presentaron ITU tuvieron un mayor tiempo de hospitalización (siete días, rango intercuartil 4-11 días) que las que no tuvieron ITU (3 días, rango intercuartil 3-4 días), asociación que fue estadísticamente significativa $(p<0,001)$. También se observó que las mujeres con incisión MIU presentaban
Tabla 4. Factores asociados a infección del tracto urinario poscesárea electiva en el INMP, 2004-2012.

\begin{tabular}{|c|c|c|c|c|}
\hline & \multicolumn{2}{|c|}{$\begin{array}{l}\text { Infección } \\
\text { del tracto } \\
\text { urinario }\end{array}$} & \multirow[t]{2}{*}{ OR } & \multirow[t]{2}{*}{ [IC 95\%] } \\
\hline & Sí & No & & \\
\hline \multicolumn{5}{|l|}{ Estado de infección } \\
\hline VIH (+) & 17 & 220 & 2,98 & {$[1,15-7,68]$} \\
\hline VIH (-) & 6 & 231 & 1 & \\
\hline \multicolumn{5}{|l|}{ Consumo de drogas } \\
\hline Sí & 1 & 16 & 1,12 & {$[0,14-8,85]$} \\
\hline No & 22 & 395 & & \\
\hline \multicolumn{5}{|l|}{ Estado nutricional } \\
\hline Desnutrido & 2 & 30 & 1,65 & {$[0,33-8,14]$} \\
\hline Eutrófico & 8 & 198 & 1 & \\
\hline Sobrepeso & 6 & 138 & 1,08 & {$[0,37-3,17]$} \\
\hline Obeso & 3 & 47 & 1,58 & {$[0,40-6,18]$} \\
\hline \multicolumn{5}{|l|}{ Uso de catéter urinario } \\
\hline Sí & 12 & 324 & 0,22 & {$[0,02-1,99]$} \\
\hline No & 1 & 6 & 1 & \\
\hline \multicolumn{5}{|l|}{ Tipo de incisión } \\
\hline Media infraumbilical & 6 & 56 & 2,49 & {$[0,94-6,58]$} \\
\hline Pfannesteil & 17 & 395 & 1 & \\
\hline \multicolumn{5}{|l|}{ Uso de antibióticos } \\
\hline No & 1 & 100 & 0,16 & {$[0,02-1,20]$} \\
\hline Sí & 22 & 351 & 1 & \\
\hline \multicolumn{5}{|l|}{ Multipara } \\
\hline Sí & 6 & 99 & 1,25 & {$[0,48-3,27]$} \\
\hline No & 17 & 352 & 1 & \\
\hline \multicolumn{5}{|l|}{ Líquido amniótico } \\
\hline Meconial & 4 & 59 & 1,40 & {$[0,46-4,25]$} \\
\hline Claro & 19 & 392 & 1 & \\
\hline
\end{tabular}

mayor probabilidad de tener una ITU que las que tuvieron incisión Pfannesteil, sin embargo dicha asociación no alcanzó significancia estadística (OR: 2,5; IC 95\%: 0,96,6). (Tabla 4).

En el grupo de mujeres VIH positivas se observó que las mujeres que no estaban en tratamiento antirretroviral tenían mayor riesgo de tener una complicación infecciosa (OR: 3,4 IC95\% [1,4-8,0]) y de fiebre sin foco aparente (OR: 5,0 IC95\% [1,1-23,2]). No se observó asociación de las complicaciones infecciosas de manera global ni individual con una carga viral detectable, el autoreporte de haber perdido una dosis de ARV, ni un estado inmunológico disminuido, tanto por categoría clínica como inmunológica de la OMS (Tabla 5).

En el modelo de regresión logística condicional stepwise backward se incluyeron las variables edad, grado de instrucción, tipo de incisión y estado de infección por el VIH para determinar su asociación con la presencia de complicaciones infecciosas. El modelo mantuvo solo una variable, el tipo de incisión, la cual continuó estando asociada a las complicaciones infecciosas (ORa: 2,3 IC95\% [1,1-4,5], p=0,019). En el caso de la 
Tabla 5. Asociación de complicaciones infecciosas y características clínicas en pacientes VIH positivas a quienes se realizó cesárea electiva en el INMP, 2004-2012.

\begin{tabular}{lccccc}
\hline & $\begin{array}{c}\text { Complicación } \\
\text { infecciosa } \\
\text { OR [IC 95\%] }\end{array}$ & $\begin{array}{c}\text { Infección de } \\
\text { tracto urinario } \\
\text { OR [IC 95\%] }\end{array}$ & $\begin{array}{c}\text { Infección de } \\
\text { herida operatoria } \\
\text { OR [IC 95\%] }\end{array}$ & $\begin{array}{c}\text { Endometritis } \\
\text { OR [IC 95\%] }\end{array}$ & $\begin{array}{c}\text { Fiebre sin foco } \\
\text { aparente } \\
\text { OR [IC 95\%] }\end{array}$ \\
\hline No TARGA & $3,38[1,43-8,00]$ & $2,83[0,93-8,63]$ & $3,34[0,95-11,82]$ & $*$ & $4,95[1,06-23,22]$ \\
Carga viral detectable & $2,71[0,81-9,07]$ & $5,16[0,73-36,39]$ & $2,15[0,46-9,99]$ & $6,36[0,25-159,83]$ & $*$ \\
CD4 2 200cel/ml & $2,23[0,66-7,54]$ & $6,21[0,88-44,04]$ & $*$ & $7,57[0,30-190,85]$ & $2,49[0,25-24,87]$ \\
Categoría OMS 4 & $1,34[0,22-8,10]$ & $*$ & $4,09[0,63-26,57]$ & $*$ & $*$ \\
Perdida dosis de ARV & $0,63[0,13-3,04]$ & $3,62[0,36-36,39]$ & $0,88[0,09-8,83]$ & $4,63[0,22-99,60]$ & $*$ \\
\hline
\end{tabular}

* No hubo casos con el factor de exposición que desarrollaran la complicación infecciosa. ARV: antirretroviral.

variable infección del tracto urinario, se incluyeron en el modelo a las variables edad, infección por el VIH, tipo de incisión y tiempo operatorio; el estado de infección por el VIH continuó asociado a la ITU (ORa: 4,5 IC95\% $[1,4-14,5], p=0,013)$, al igual que el tiempo operatorio $(p=0,021)$.

\section{DISCUSIÓN}

El parto por cesárea es indiscutiblemente beneficioso para la disminución de la TV ${ }^{(5-6)}$ en las mujeres con $\mathrm{VIH}$ en general, lo que ha generado un incremento del número de las mismas en pacientes VIH positivas, quienes estarían a su vez en un mayor riesgo de complicaciones debido al compromiso de su estado inmunológico. Si bien la mayoría de estudios perinatales en pacientes VIH se han enfocado a la disminución de la transmisión vertical, estudios más recientes ${ }^{(13-15)}$, como el nuestro, buscan evaluar las consecuencias maternas de dichas intervenciones en términos de morbilidad, sobre todo considerando que el beneficio en mujeres con cargas virales indetectables es menor.

En el presente estudio encontramos una incidencia de complicaciones infecciosas en mujeres $\mathrm{VIH}$ reactivas de $14 \%$, similar a lo descrito por Moodliar et al (19) y por Louis et al (20). Sin embargo no encontramos asociación significativa entre el estado de infección por el VIH y la presencia de $\mathrm{Cl}$, a diferencia de estudios previos ${ }^{(21,22)}$. Debemos considerar que en dichos estudios combinaban las complicaciones infecciosas con anemia leve, y la mayor parte de la asociación correspondía a dicha enfermedad, la cual ha sido ampliamente asociada al uso de antirretrovirales (23). Igualmente algunos estudios incluían participantes de mayor riesgo como usuarias de drogas endovenosas o mujeres que no habían tenido controles prenatales. Por otro lado encontramos también, en nuestra revisión de la literatura, otros estudios que no encontraron asociación entre el estado de infección por el VIH y las complicaciones infecciosas poscesárea ${ }^{(24-27)}$.
A pesar de no encontrar asociación global con las complicaciones infecciosas, sí encontramos asociación con infección del tracto urinario, similar a lo informado por otros autores ${ }^{(13,21)}$. La presencia de ITU estuvo además asociada a un mayor tiempo operatorio, lo que se podría explicar por un mayor tiempo de exposición al catéter urinario.

En nuestro estudio no se encontró asociación entre el estado de infección por el VIH y la endometritis, observación también realizada por otros investigadores ${ }^{(21-26)}$. Igualmente en el caso de fiebre sin foco aparente ${ }^{(25-27)}$; y de infección de herida operatoria ${ }^{(18-25)}$.

Otros estudios ${ }^{(25,27)}$ han descrito asociación entre niveles inferiores de CD4 y presencia de complicaciones postparto, asociación que no fue observada en nuestro estudio. Sin embargo, a diferencia de Ferrero et al. ${ }^{(13)}$ sí encontramos asociación entre el no recibir TARGA y $\mathrm{Cl}$.

No es de extrañar que hubiera asociación entre las $\mathrm{Cl}$ y un mayor tiempo de hospitalización, ya que aquellas mujeres con una complicación deben permanecer más tiempo hasta que esta sea diagnosticada y controlada. Otros estudios han encontrado también asociación entre un mayor tiempo operatorio y $\mathrm{Cl}^{(21,24)}$.

El bajo número de complicaciones infecciosas encontrado en ambos grupos puede deberse en parte al uso rutinario de profilaxis antibiótica, según guías clínicas del INMP, así como a la ausencia de factores de riesgo como ruptura de membranas o inicio de trabajo de parto. Además, en el caso de las mujeres $\mathrm{VIH}$ reactivas, la mayoría se encontraba en un estado clínico e inmunológico leve, con cargas virales indetectables y usando ARV. Cabe destacar también que ambos grupos fueron manejados de manera similar, con igual proporción de uso de antibióticos, catéter urinario y tiempo operatorio, entre otros.

A raíz de los resultados se podría sugerir que las mujeres VIH positivas se beneficiarían de un curso más prolongado de antibióticos debido a su mayor riesgo de 
complicaciones infecciosas; sin embargo, Turnquest et al. (28) no encontraron diferencia en el riesgo de endometritis entre una dosis única de antibiótico y un curso más prolongado.

Entre las limitaciones del estudio tenemos que el personal de salud responsable de la atención de las pacientes estaba al tanto de su estado de infección, lo que podría haber condicionado un manejo diferenciado entre grupos; sin embargo, se pudo observar que el uso de antibióticos y de catéter urinario, así como el tiempo operatorio fue similar en los grupos de mujeres expuestas y no expuestas. De igual manera, ambos grupos fueron bastante similares y las características en las que difieren no demostraron estar relacionadas con las complicaciones infecciosas. Sin embargo, sí podría haber generado que las mujeres HIV positivas se siguieran más de cerca con la finalidad de detectar tempranamente cualquier posible complicación.

Debemos considerar que el no haber encontrado diferencia de la frecuencia de $\mathrm{Cl}$ entre los grupos expuestas y no expuestas podría deberse a que las frecuencias observadas fueron menores a las esperadas, y en base a las que se calculó el tamaño muestral, lo cual disminuye la potencia del estudio.

Si bien los presentes resultados no están en contra de las recomendaciones actuales del parto por cesárea electiva en mujeres VIH-positivas, consideramos que sí deberían ser parte de la consejería a la paciente en relación a la vía para finalizar el embarazo. El riesgo elevado de morbilidad materna y la posibilidad de transmisión vertical del VIH deben ser sopesados, particularmente cuando la gestante están en TARGA y con cargas virales indetectables.

En conclusión, no se encontró diferencia significativa en la incidencia de complicaciones infecciosas de las cesáreas electivas, entre mujeres $\mathrm{VIH}$ reactivas y no reactivas; sin embargo las mujeres infectadas por el virus del VIH tienen mayor riesgo de presentar infecciones del tracto urinario después de una cesárea electiva. El principal factor de riesgo asociado a complicación infecciosa poscesárea electiva fue el tipo de incisión y el tiempo operatorio a una infección del tracto urinario.

Contribuciones de autoría: RT y CG participaron en la concepción y diseño del artículo; RT en la recolección y obtención de resultados, análisis e interpretación de datos, así como en la redacción del artículo, LH y CG participaron en la asesoría estadística; CV y CL brindaron asesoría técnica o administrativa y han participado en la captación de pacientes. Todos los autores participaron en la revisión crítica del artículo y aprobación de su versión final.

Fuentes de financiamiento: autofinanciado.

Conflictos de interés: los autores declaran no tener ningún conflicto de interés.

\section{REFERENCIAS BIBLIOGRÁFICAS}

1. World Health Organization. Global report: UNAIDS report on the global AIDS epidemic 2010. Geneva: WHO; 2010.

2. Dirección General de Epidemiología. Situación del VIH/SIDA en el Perú. Boletín Epidemiológico mensual [Internet]. 2012;(6) [citado el $10 \mathrm{de}$ septiembre del 2012]. Disponible en: http://www.dge.gob.pe/vigilancia/vih/ Boletin_2012/julio.pdf

3. Pun M, Escudero A, Suárez LA, Alvarado R; Unidad Técnica de Vigilancia, Investigación y Análisis de la ITS, VIH-SIDA. Análisis de la situación de la epidemia del VIH-SIDA [Internet]. Lima: MINSA; 2011 [citado el 10 de septiembre del 2012]. Disponible en: http://www.dge. gob.pe/notas_prensa/2011/ResumenSit_VIH2011_01Dic.pdf

4. The mode of delivery and the risk of vertical transmission of human immunodeficiency virus type 1--a meta-analysis of 15 prospective cohort studies. N Engl J Med. 1999;340(13):977-87.

5. Mofenson LM. Successes and challenges in the perinatal HIV-1 epidemic in the United States as illustrated by the HIV-1 Serosurvey of childbearing women. Arch Pediatr Adolesc Med. 2004;158(5):422-5.

6. Caesarean section and risk of vertical transmission of HIV-1 infection. The European Collaborative Study. Lancet. 1994:343(8911):1464-7.

7. Chen KT, Sell RL, Tuomala RE. Costeffectiveness of elective cesarean delivery in human immunodeficiency virusinfected women(1). Obstet Gynecol. 2001;97(2):161-8.

8. Ministerio de Salud del Perú. Norma técnica para la prevención de la transmisión vertical (Madre-Niño) del VIH. NT $\quad \mathrm{N}^{\circ}$-2008-MINSA/DGSP-V.01. Lima: MINSA; 2008.

9. Read JS, Newell ML. Efficacy and safety of cesarean delivery for prevention of mother- to-child transmission of HIV-1. Cochrane Database Syst Rev. 2005;(4):CD005479.

10. Gregory KD, Jackson S, Korst L, Fridman M. Cesarean versus vaginal delivery: whose risks? Whose benefits? Am J Perinatol. 2012;29(1):7-18.

11. Hadar E, Melamed N, TzadikevitchGeffen K, Yogev Y. Timing and risk factors of maternal complications of cesarean section. Arch Gynecol Obstet. 2011;283(4):735-41.

12. Semprini AE, Castagna C, Ravizza M, Fiore S, Savasi V, Muggiasca ML, et al. The incidence of complications after caesarean section in $156 \mathrm{HIV}$-positive women. AIDS. 1995;9(8):913-7.

13. Ferrero S, Bentivoglio G. Post-operative complications after caesarean section in HIV-infected women. Arch Gynecol Obstet. 2003;268(4):268-73.

14. Fiore S, Newell ML, Thorne C; European HIV in Obstetrics Group. Higher rates of post-partum complications in HIV- 
infected than in uninfected women irrespective of mode of delivery. AIDS. 2004;18(6):933-8.

15. Griffiths J, Demianczuk N, Cordoviz M, Joffe AM. Surgical site infection following elective Caesarian section: a case-control study of postdischarge surveillance. J Obstet Gynaecol Can. 2005;27(4):340-4.

16. Watts DH, Lambert JS, Stiehm ER, Bethel J, Whitehouse J, Fowler MG, et al. Complications according to mode of delivery among human immunodeficiency virus-infected women with CD4 lymphocyte counts of $<$ or $=500 / \mathrm{microL}$. Am J Obstet Gynecol. 2000;183(1):100-7.

17. Urbani G, de Vries MM, Cronjé HS, Niemand I, Bam RH, Beyer E. Complications associated with cesarean section in HIV-infected patients. Int J Gynaecol Obstet. 2001;74(1):9-15.

18. NIH Consensus Development Task force statement on cesarean childbirth. Am J Obstet Gynecol. 1981;139(8):902-9.

19. Moodliar S, Moodley J, Esterhuizen TM. Complications associated with caesarean delivery in a setting with high HIV prevalence rates. Eur J Obstet Gynecol Reprod Biol. 2007;131(2):138-45.

20. Louis J, Buhari MA, Allen D, Gonik B, Jones TB. Postpartum morbidity associated with advanced HIV disease. Infect Dis Obstet Gynecol. 2006;2006:79512.

21. Lapaire O, Irion $\mathrm{O}$, Koch-Holch A, Holzgreve W, Rudin C, Hoesli l, et al. Increased peri- and post-elective cesarean section morbidity in women infected with human immunodeficiency virus-1: a case-controlled multicenter study. Arch Gynecol Obstet. 2006;274(3):165-9.

22. Rodriguez EJ, Spann C, Jamieson D, Lindsay M. Postoperative morbidity associated with cesarean delivery among human immunodeficiency virus-seropositive women. Am J Obstet Gynecol. 2001;184(6):1108-11.

23. Zuk DM, Hughes CA, Foisy MM, Robinson JL, Singh AE, Houston S. Adverse effects of antiretrovirals in HIV-infected pregnant women. Ann Pharmacother. 2009;43(6):1028-35.

24. Louis J, Landon M, Gernoviez RJ, Leveno KJ, Spong CY, Rouse DJ, et al. Perioperative morbidity and mortality among human immunodeficiency virus infected women undergoing cesarean delivery. Obstet Gynecol. 2007;110(2 Pt 1):385-90.

25. Vimercati A, Greco P, Loverro G, Lopalco PL, Pansini V, Selvaggi L. Maternal complications after caesarean section in HIV infected women. Eur J Obstet Gynecol Reprod Biol. 2000;90(1):73-6.

26. Grubert TA, Reindell D, Kästner R, LutzFriedrich R, Belohradsky BH, Dathe O. Complications after caesarean section in HIV-1-infected women not taking antiretroviral treatment. Lancet. 1999;354(9190):1612-3.

27. Grubert TA, Reindell D, Kästner R, Belohradsky BH, Gürtler L, Stauber M, et al. Rates of postoperative complications among human immunodeficiency virus-infected women who have undergone obstetric and gynecologic surgical procedures. Clin Infect Dis. 2002;34(6):822-30.

28. Turnquest MA, How HY, Cook CR, O’Rourke TP, Cureton AC, Spinnato JA, et al. Chorioamnionitis: is continuation of antibiotic therapy necessary after cesarean section? Am J Obstet Gynecol. 1998;179(5):1261-6.

Correspondencia: Romina Tejada

Dirección: Calle José Santos Chocano 199 Bellavista, Instituto de Medicina Tropical "Daniel Alcides Carrión".

Teléfono: (511) 4525259

Correo electrónico:kurotani@gmail.com

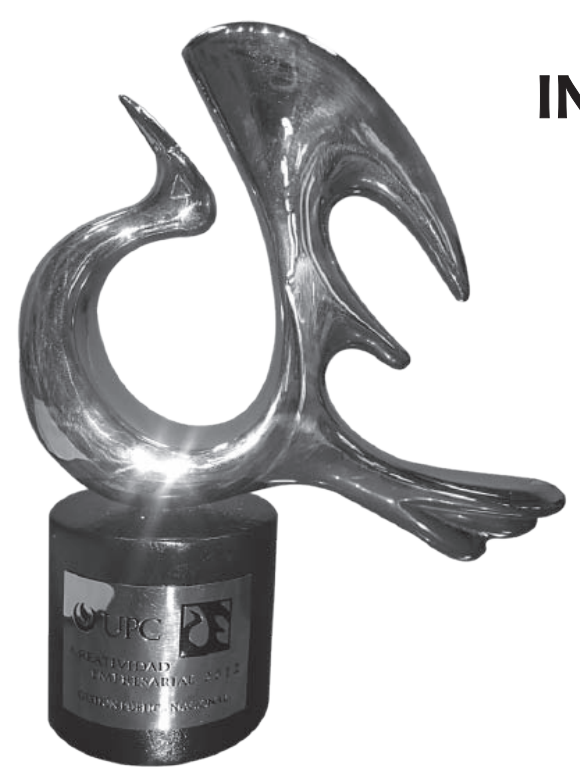

\author{
Ganadores en el concurso \\ Creatividad Empresarial 2012
}

Premio en la categoría

GESTIÓN PÚBLICA NACIONAL

Kit Tariki-Dengue: Acercando el Diagnóstico

de Dengue en Comunidades más Necesitadas

Investigamos para proteger la salud 